\title{
Bemerkung zur Axiomatik der Größen und Mengen.
}

\author{
Von
}

A. Schoenflies in Frankfurt a. M.

In dem Artikel ,Zur Axiomatik der Mengenlehre" "1) habe ich die Axiome, die sich mit den Gebieten der Äquivalenz, der Mengenteilung und Mengenvergleichung beschäftigen, einer Frörterung unterzogen. An zwei Resultate dieses Artikels knüpfe ioh hier an. Erstens einmal, da die in ihm durchgeführten Untersuchungen auf die Elemente der Mengen gar nicht eingehen, so stellen sie, allgemein gesprochen, axiomatische Betrachtungen über Größen und Größenbeziehungen dar, an denen die Mengen ja Teil haben; und zweitens hatte eine der dort analysierten Beziehungen den Gedanken nahegelegt, auch Größen entgegengesetzter Art (resp. Mengen von zweierlei Art von Elementen) in Betracht zu ziehen, und auf sie die oben genannten Operationen auszudehnen. Hierzu gebe ich im folgenden einige Ergänzungen.

Berëeits a. a. O. war bemerkt worden, daß es naturgemäß der Untersuchung bedarf, ob für die so charakterisierten Mengen die weiteren allgemeinen Sätze der Cantorschen Theorie in Kraft bleiben. Inzwischen hat mir Herr A. Fränkel mitgeteilt, da $\mathrm{B}$ für das von mir konstruierte Beispiel schon ein Teil der in meinem Artikel zugrunde gelegten Axiome versagt; und zwar ein Teil der Axiome über Teilmengen. thber Teilmengen habe ich zwei Axiome an die Spitze gestellt. Wird die Beriehung, dab $M^{\prime}$ Teilmenge von $M$ ist, durch

\section{$M^{\prime} t M$}

bezeichnet, so lauten diese Axiome:

I. Aus $M^{\prime} t M$ und $M^{\prime \prime} t M^{\prime}$ folgt $M^{\prime \prime} t M$ (der assoziative Charakter des Teilmengenbegriffs).

II. Jede Teilmenge $M^{\prime}$ von $M$ bestimmt eindeutig eine aweite Teilmenge $\grave{M}_{1}$ von $M$, die ihre Komplementärmenge bezïglich $M$ heißt.

1) Amsterdam Ac. Proc. 22 (1920); abgedruckt in den Math. Ann. 88 (1921), S. 173. 
Von diesen grundlegenden Axiomen versagt für das a. a. 0 . behandelte Beispiel das zweite; das erste bleibt-bestehen. Das Beispiel war so erdacht, daß man als Größen einseitig begrenzte Geraden von unendlicher Länge, aber von zwei entgegengesetzten Richtungen wählte, als Teilmenge jeden ebenfalls unendlichen Bestandteil zuläBt und die Aquivalenz z. B. durch eindentige Āhnlichkeitsabbildung definiert. In der Tat bleibt dann das erste Axiom erhalten, das zweite aber nicht. Wenn man nämlich von einer solchen Geraden eine Teilmenge $M^{\prime}$ im obigen Sinne abspaltet, so bleibt außerdem noch ein endlicher Abschnitt übrig, und der entspricht unserm Begriff der Teilmenge nicht mehr. Das nämliche gilt fïr das zweite a. a. 0 . behandeite Beispiel.

Diese von Herm Fränkel bemerkte Tatsache ist auch deshalb von Interesse, weil sie den Teilmengenbegriff und seine Bhigenart in neuer Weise beleuchtet. Sie zeigt nämlich in erster Linie die Unabhängigkeit der Axiome I und II, sie zeigt außerdem, daß der Teilmengenbegriff gewisse mathematische Operationen auch dann noch zuläBt, wenn das Komplementärmengenaxiom nicht exfüllt ist.

Ioh erinnere weiter daran, daß das vorstehende Beispiel zu folgendem Zweoke erdacht war. Ich hatte a. a. O. das Axiom aufgestellt,

$$
\text { aus } M d N \text { und } N d P \text { folge } M d P, 2)
$$

und es sollte durch das Beispiel belegt werden, daß man Größen oder Mengen einführen könne, die den Aquivalenzaxiomen usw. folgen, für die aber das eben genannte Axiom nicht exfüllt ist. Diesen Zweck kann man durch eine einfache Abänderung des Beispiels erreichen. Es genügt dazu, auch den endlichen Bestandteil $\boldsymbol{M}_{1}$, der durch Abtrennung der Teilmenge $\boldsymbol{M}^{\prime}$ von $\boldsymbol{M}$ übrigbleibt, als Teilmenge von $M$ zurulassen und die Äquivalenz wieder auf eine Āhnlichkeitstrensformation und die Ubereinstimmung der Richtung zu gründen.

Un die GröBenklasse, die sich so érgibt, noch genaues zar umschreiben, wollen wir festsetzen, daB

1. alle betrachteten Strecken auf Geraden liegen, die parallel einer $x$-Achse verlaufen;

2. die Endpunkte der Strecken rationslo Absrissen haben sollen ${ }^{8}$ );

3. nur alle diejenigen parallelen Geradon als Träger von Strecken in Betracht kommen, die eine $y$-Achse in rationalen/Punkten schneiden, so daß , beiläufig bemerkt, unsere Größenklasse abzählbar ist. Auf die Gesamtheit der so eingefiuhrten Strecken wollen wir nun die Teilmengenaxiome übertragen. Zunächst ist klar, daß hier die Axiome I und II beide erfiillt sind. Das gleiche

9) $M d N$ bedeutet: Es gibt weder eine Teilmenge ron $M$, die äguivalent $N$ ist, noch eine Teilmenge von $N$, die âquivalent $M$ iat.

9) Man hat die Aquivalenz alsdenn auf ahnliohe Abbildung mit rationalen Kaeffizionten zu gründen. 
gilt aber auch noch von den folgenden a. a. 0 . über Teilmengen aufgestellten Axiomen:

III. Die Komplementärmenge von $M_{1}$ ist wiederum $M^{\prime}$.

IV. Die beiden Komplementärmengen $M^{\prime}$ und $M_{1}$ einer Menge $M$ sind fremde Mengen (d. h. ohne gemeinsame Teilmenge).

Bestehen bleibt außerdem auch das Axiom $I$, von $\S 3$, nämlich

$V$. Ist $M^{\prime} t M$ und $M \sim N$, so folgt daraus notwendig die Existenz einer Menge $N^{\prime}$, für die zugleich gilt

$$
N^{\prime} t N \text { und } M^{\prime} \sim N^{\prime},
$$

und dies ist dasjenige Axiom, das für die gesamten Beweisführungen an vorderster Stelle steht.

Nicht bestehen dagegen bleibt das Axiom $\mathrm{V}$ von $\$ 2$, daß nämlich zwei fremde Mengen $P$ urd $Q$ stets eine und nur eine Menge $M$ bestimmen, deren Komplementärmengen sie sind. (Es versagt z. B., wenn die beiden Mengen $P$ und $Q$ auf verschiedenen Geraden liegen.) Es ist auch klar, dab diesem Axiom, das in der Mengenlehre die Erzeugung einer neuen Menge aus zwei gegebenen fordert, also ein Existenzaxiom ist, eine wesentlich andere Stellung zukommt wie den vorstehenden").

.: Für die Beziehungen zweier. Strecken der so umschriebenen Größenklassen liegen folgende Möglichkeiten vor: Man kann zwei unendliche Strecken, zwei endliche, sowie eine endliche und eine unendliche, miteinander vergleichen. Wir fassen zuerst den Fall ins Auge, daß beide Strecken gleichgerichtet sind. Man hat dann die vier Möglichkeiten
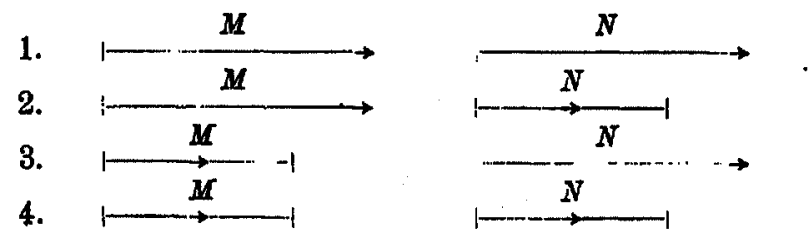

und hat im ersten und vierten Fall offẹpbar die Beriehung

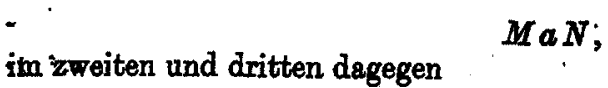

im zweiten und dritten dagegen

$$
\text { - } M B N \text {, oder } M c N \text {. }
$$

Für Strecken einer und derselben Richtung ist also der Fall $M d N$ überhaupt ausgeschlossen, genau wie für die Klasse der unendlichen Mengen.

4) Von meinem hiosigen Kollegen Hellinger wurde ich dareuf aufmerksam gomacht, daß man gewirse Mengen von Strecken als Objekto -so einfübren kann, daß für aie das obige Existenzaxiom ebenfalls in Kraft bleibt. 
Geht man zu zwei Strecken $M$ und $N$ entgegengesetzter Richtung über, so ist klar, daß für sie offenbar in jedem Fall die Beziehung

\section{$M d N$}

erfiullt ist, da ja die Aquivalenz auch die tbereinstimmung der Richtungen einschließen soll.

Wir prïfen nunmehr, welche Folgerungen sich aus

\section{$M d N$ und $N d P$}

in den einzelnen Fällen ergeben. Gemäß dem Vorstehenden kann die Beziehung - $M d N$ auf folgende vier Arten realigiert sein
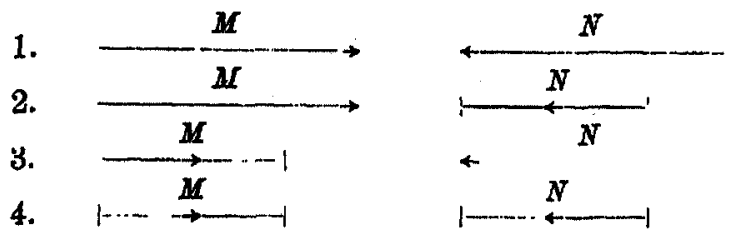

und analog haben wir fïr $N d P$ die Möglichkeiten
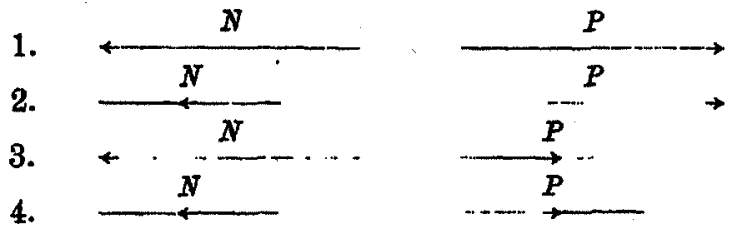

Wir können dann zunächst kombinieren 1 mit 1, 2 mit 2, 3 mit 3 und 4 mit 4, und erhalten in allen Fällen die Beriehung

$$
\operatorname{MaP} \text {; }
$$

ebenso können wir kombinieren 1 mit 3, 2 mit 4, 3 mit 1, 4 mit 2, und finden hierfür die Beriohungen

$$
M b P \text { und } M C P \text {, }
$$

nämlich $M b P$ fïr (13) und (24) und $M C P$ ffir (31) und (42). In keinem Falle aber erhalten wir

oder kürzer ausgedrückt:

$$
M d P
$$

Aus $(d d)$ folgt $a$ oder $b$ oder $c$, aber niemals $d$.

Damit ist der obengenannte Zweok erreicht. Unser Beispiel genügt den sämtlichen oben über Teilmengen aufgestellten Axiomen und zeigt durch seine Eigenart wiederum den axiomatischen Charakter der a. a. 0 . aufgestellten und oben wiederholten Forderung D \%

6) Die Frage, welobe anderen Folgerungen aus den in $\$ 2$ (a.a.0.) betrachteten Verkmüpfungen sich ziehon lassen, mag bier auBer Betracht bleiben. 
Unser Resultat ist noch aus einem andern Grunde bemerkenswert. Es nimmt einer von mir a. a. 0 . benutzten SchluBwendung die Beweiskraft. Ich habe nämlich dort ( $\$ .181$ ) behauptet, daß aus den Prämissen

\section{$M d N$ und $N d P$}

weder $M b P$ noch $M C P$ folgen könne, und zwar auf Grund folgender Erwägung. Die Beziehungen $M d N$ und $N d P$ können auch in die Form

\section{$N d M$ und $P d N$}

geschrieben werden. Würde nun aus $M d N$ und $N d P$ der Schluß $M b P$ möglich sein, so müßte wegen der vorstehenden umgekehrten Schreibweise auch der Schluß $P b M$ möglich sein; aber $M b P$ und $P b M$ widersprechen einander.

Dieser eben wieder benutzte, aber offenbar unzulässige Schluß galt mir damals als evident. Worin liegt die Frklärung? In dem Tatbestand, auf den ich a. a. O. gerade mit allem Nachdruck hingewiesen habe, daß man nämlich aus rein negativen Behauptungen ohne weiteres überhaupt keinen SchluB ziehen dürfe - was aber eben doch wieder geschehen ist. Die obige Umkehr der Schlußweise hat nämlich nur eine formale, also inhaltlose Bedeutung. Die Beziehungen $M d N$ and $N d P$ sagen zwar in rein negativer Hinsicht dasselbe aus: aber die Objekte $\boldsymbol{M}$ und $\boldsymbol{N}$ brauchen in die negative Beziehung $M d N$ keineswegs inhaltlich gleichwertig oder symmetrisch einzugehen. Wenn sie also benutzt werden können oder sollen, um aus ihnen und einer gleichfalls negativen Beziehung $\boldsymbol{N} d \boldsymbol{P}$ eine Beziehung zwischen $\boldsymbol{M}$ und $\boldsymbol{P}$ abzuleiten, so kann sehr wohl die Sonderart der Objekte $M$ und $N$ sowie $N$ und $P$ für die resultierende Beziehung bestimmend in Frage kommen. Oder anders ausgedrückt: Fin rein negatives Verhältnis zwischen zwei Objekten $M$ und $\boldsymbol{N}$ kann durch die Bigenart von $\boldsymbol{M}$ und $N$ sehr mannigfach begründet sein, und gerade deshalb versagt bei der Kombination zweier solcher Beziehungen die Möglichkeit logischer Folgen, und macht also, falls eindeutige Frolgen gezogen verden sollen, deren axiomatische Aufstellung nötig.

(Elingegaxigen am 15. 8. 1921.) 